\title{
Langmuir Wave energy upconversion in presence of Lower Hybrid Wave turbulence in Polar Ionospheric Plasma
}

\author{
S.J. Gogoi ${ }^{*}$, P.N. Deka ${ }^{2}$ \\ ${ }^{1}$ Department of Physics, Tinsukia College, Tinsukia, Assam, India \\ ${ }^{2}$ Department of Mathematics, Dibrugarh University, Dibrugarh, Assam, India \\ *Correspondence author: satyajyotitsk@gmail.com
}

Available online at: www.isroset.org

Received: 07/Jun/2019, Accepted: 19/Jun/2019, Online: 30/Jun/2019

\begin{abstract}
In the Earth's ionosphere wide range of instabilities are observed due to presence of free energy sources. Wave-wave and wave-particle interaction processes may take place here. Through wave-particle interaction process wave energy can be pumped up from the system for amplification of high frequency non-resonant wave where low frequency turbulence is present. In this study we have considered ionosphere as homogeneous medium and wish to investigate probable amplification of nonresonant Langmuir wave in the presence of resonant Lower hybrid wave through nonlinear wave-particle interaction. We have estimate growth rate of Langmuir wave using satellite observational data.
\end{abstract}

Keywords: Ionospheric Plasma, Langmuir wave, Lower hybrid wave, Plasma maser effect

\section{INTRODUCTION}

Ionospheric plasma is found to be thermodynamically non equilibrium and from ground and space based observatories, turbulence and instabilities are observed in this region. Solar wind is the main source of energy and momenta of this open plasma system. This region supports variety of electrostatic and electromagnetic plasma waves. In space and in laboratory plasma system it is observed amplification of high frequency plasma wave for the interaction with low frequency plasma wave turbulence.

Within the framework of parametric theory, Davis [1] studied amplification of non-resonant wave but the growth rate was not large. On the basis of linear response theory, Tsytovich et. al [2] were studied growth rate of Langmuir wave in the presence of ion acoustic wave. A different type of non-linear wave-particle interaction called plasma maser effect was developed by Nambu [3] on the platform of nonlinear theory of turbulent plasma and it predicts energy up conversion (/down conversion) of non-resonant wave in the presence of resonant wave. Here, plasma maser instability arises from the interaction between particles and the nonlinear modulating electric field. This electric field occurs for the coupling between resonant and non-resonant plasma mode. For this nonlinear field the charged particles are accelerated nonlinearly to generate amplified electrostatic wave and electromagnetic radiation through modulated field.

\section{II.RELATED WORKS}

In the polar ionosphere region naturally occurring Langmuir turbulence was predicted in several studies [4, 5, 6, 7].Ergun et al. [8] discussed on the generation of modulated Langmuir wave packets in the auroral ionosphere. It was found that the frequency of electrostatic Langmuir waves in the ionosphere was affected to be by the photoelectrons or auroral secondary electrons [9].The low frequency electrostatic lower hybrid wave was detected in the ionospheric plasma and electrons were found to be accelerated by this wave along the auroral field lines [10]. Pasmanik et al.[11] studied properties of lower hybrid wave propagation in the auroral ionosphere taking inhomogeneity both along and across the Earth's magnetic field. Kelley [12] found lower hybrid wave played a vital role in ionospheric turbulence processes. Lizunov et al.[13] studied wave-wave interaction between Langmuir wave and lower hybrid wave to explain Freja satellite observational data in the polar ionosphere and found from frequency spectra both secondary Langmuir wave and lower hybrid wave were parametrically connected. Within the nonlinear wave-particle interaction framework in several studies were carried on plasma maser instabilities in both space and laboratory plasma environment associated with different physical parameter gradients [14,15,16,17].In space plasma system, in addition with free energy sources, the wave energy of non-resonant mode was found to be enhanced in presence of resonant wave. Deka [18] studied amplification of upper hybrid wave in presence of lower 
hybrid wave turbulence through plasma maser effect for the Auroral Kilometric Radiation (AKR) phenomena in magnetospheric plasma.

In this paper, we present our theoretical investigation on growth of Langmuir wave in presence of lower hybrid wave in the polar ionosphere region through plasma maser instability and estimate by using Freja satellite data.

\section{III.FORMULATION}

We consider electrostatic lower hybrid turbulence to be present in the magnetised plasma where the direction of the external magnetic field is taken in the z-direction. Here the lower hybrid wave has propagation vector $\vec{k}=\left(k_{\perp}, 0, k_{\square}\right)$ where $\perp$ and $\square$ represent perpendicular and parallel direction to the external magnetic field. We assume in this open plasma system high frequency Langmuir wave has propagation vector $\vec{K}=\left(0,0, K_{\square}\right)$ along the direction of the magnetic field. In this problem it is neglected the temperature variation and their anisotropy.

For this system the zero order particle distribution function for $\mathrm{j}^{\text {th }}$ species is considered as

$$
\left.f_{0 j}(\vec{v})=\left(\frac{m_{j}}{2 \pi T_{j}}\right)^{\frac{3}{2}} e^{\left[-\frac{m_{j}}{2 T_{j}}\left\{v_{j \perp}^{2}+v_{j \jmath}^{2}\right\}\right.}\right]
$$

here ‘j’ refers electron (e) and ion(i).

\section{IV.MATHEMATICAL ANALYSIS}

The interaction of non-resonant Langmuir wave with resonant lower hybrid wave is governed by the VlasovPoisson system of equations

$$
\left[\frac{\partial}{\partial t}+\vec{v}_{j} \cdot \frac{\partial}{\partial \vec{r}}-\frac{e_{j}}{m_{j}}\left(\vec{E}+\frac{\vec{v}_{j} \times \vec{B}}{c}\right) \cdot \frac{\partial}{\partial \vec{v}_{j}}\right] F\left(\vec{r}, \vec{v}_{j}, t\right)=0
$$

and

$$
\vec{\nabla} \cdot \vec{E}=-4 \pi n_{j} e_{j} \int f\left(\vec{r}, \vec{v}_{j}, t\right) \cdot d \vec{v}
$$

The unperturbed distribution function for $\mathrm{j}^{\text {th }}$ species and the unperturbed electric field and magnetic field are taken as

$$
F_{0 j}=f_{0 j}+\varepsilon f_{1 j}+\varepsilon^{2} f_{2 j}
$$

$\vec{E}_{0 l}=\varepsilon \vec{E}_{l}+\varepsilon^{2} \vec{E}_{2}$

$\vec{B}_{l}=\vec{B}_{0}$

where $f_{0 j}$ is the space and time averaged part of the distribution function, $f_{1 j}$ and $f_{2 j}$ are the fluctuating parts due to the low-frequency lower hybrid turbulence, $\varepsilon$ is the ordering of the low-frequency turbulence, $\vec{E}_{l}$ is the lower hybrid turbulent field, $\vec{E}_{2}$ is the second order electric field and the confining magnetic field $\vec{B}_{0}$ is taken along $\mathrm{z}$ direction.

Using equation (4), equation (2) can be written as

$$
\left[\frac{\partial}{\partial t}+\vec{v}_{j} \cdot \frac{\partial}{\partial \vec{r}}-\varepsilon \frac{e_{j}}{m_{j}} \vec{E}_{l} \cdot \frac{\partial}{\partial \vec{v}_{j}}-\frac{e_{j}}{m_{j}}\left(\frac{\vec{v}_{j} \times \vec{B}}{c}\right) \cdot \frac{\partial}{\partial \vec{v}_{j}}\right]\left(f_{0 j}+\varepsilon f_{1 j}+\varepsilon^{2} f_{2 j}\right)=0
$$

To the order of $\varepsilon$ we obtain

$$
\left[\frac{\partial}{\partial t}+\vec{v}_{j} \cdot \frac{\partial}{\partial \vec{r}}-\frac{e_{j}}{m_{j}}\left(\frac{\vec{v}_{j} \times \vec{B}}{c}\right) \cdot \frac{\partial}{\partial \vec{v}_{j}}\right] f_{1 j}=\frac{e_{j}}{m_{j}} \vec{E}_{l} \cdot \frac{\partial}{\partial \vec{v}_{j}} f_{0 j}
$$

Applying Fourier transform and the method of integration along unperturbed orbit [19] under the assumption $E_{l \square} \square E_{\perp}$, we obtain from equation (6) as

$$
f_{1 j}(\vec{k}, \omega)=-i \sum \frac{e_{j}}{m_{j}} E_{l \square} \frac{\partial f_{0 j}}{\partial v_{j \square}} \Pi_{a, b}
$$

where

$$
\Pi_{a, b}=\frac{\sum_{a, b} j_{a}\left(\frac{k_{\perp}{ }^{v} j \perp}{\Omega_{j}}\right) j_{b}\left(\frac{k_{\perp}{ }^{v} j \perp}{\Omega_{j}}\right) e^{[i(b-a) \theta]}}{\left(a \Omega_{j}+k_{\perp} v_{j \square}-\omega\right)+i 0^{+}}
$$

Here we are using cylindrical co-ordinates are

$$
v_{x}=v_{\perp} \cos \theta, v_{y}=v_{\perp} \sin \theta, v_{z}=v_{\square}
$$

and transformation equations are 


$$
\begin{aligned}
& \frac{\partial}{\partial v_{x}}=\cos \theta \frac{\partial}{\partial v_{\perp}}-\frac{\sin \theta}{v_{\perp}} \frac{\partial}{\partial \theta} \\
& \frac{\partial}{\partial v_{y}}=\sin \theta \frac{\partial}{\partial v_{\perp}}+\frac{\cos \theta}{v_{\perp}} \frac{\partial}{\partial \theta} \\
& \frac{\partial}{\partial v_{z}}=\frac{\partial}{\partial v_{\square}}
\end{aligned}
$$

and the unperturbed particle orbits are

$$
\begin{aligned}
& x^{\prime}-x=-\frac{v_{\perp}}{\Omega_{j}} \sin \left(\theta-\Omega_{j} \tau\right)+\frac{v_{\perp}}{\Omega_{j}} \sin \theta \\
& y^{\prime}-y=\frac{v_{\perp}}{\Omega_{j}} \cos \left(\theta-\Omega_{j} \tau\right)-\frac{v_{\perp}}{\Omega_{j}} \cos \theta \\
& z^{\prime}-z=v_{\sqsubset} \tau
\end{aligned}
$$

where

$$
\tau=t^{\prime}-t
$$

Let resonant lower hybrid turbulence has wave field as $\vec{E}_{l}=\left(E_{l \perp}, 0, E_{l \square}\right)$ and to this quasisteady state we apply perturbation wave field $\mu \delta \vec{E}_{h}$ of high frequency Langmuir wave field $\mu \delta \vec{E}_{h}=\left(0,0, \delta E_{h}\right)$ where $\varepsilon \square \mu$.For this perturbation the total perturbed particle distribution function, the electric field and the magnetic field are

$$
\begin{aligned}
& \delta f_{j}=\mu \delta f_{h}+\mu \varepsilon \delta f_{l h}+\mu \varepsilon^{2} \Delta f \\
& \delta \vec{E}=\mu \delta \vec{E}_{h}+\mu \varepsilon \delta \vec{E}_{l h}+\mu \varepsilon^{2} \Delta \vec{E} \\
& \delta \vec{B}=0
\end{aligned}
$$

where $\delta f_{l h}$ and $\Delta f$ are distribution functions for the modulated fields, $\delta f_{h}$ is fluctuating part due to low frequency lower hybrid wave, $\delta \vec{E}_{l h}$ and $\Delta \vec{E}$ are modulated fields.

Let the operator

$$
\hat{L}=\frac{\partial}{\partial t}+\vec{v}_{j} \cdot \frac{\partial}{\partial \vec{r}}-\frac{e_{j}}{m_{j}}\left(\frac{\vec{v}_{j} \times \vec{B}_{0}}{c}\right) \cdot \frac{\partial}{\partial \vec{v}_{j}}
$$

Using equation (9) in Vlasov equation (2) for the perturbed state we get

$$
\left[\hat{L}-\frac{e_{j}}{m_{j}}\left(\varepsilon \vec{E}_{l}+\varepsilon^{2} \vec{E}_{2}+\mu \delta \vec{E}_{h}+\mu \varepsilon \delta \vec{E}_{h}+\mu \varepsilon^{2} \Delta \vec{E}_{h}\right) \cdot \frac{\partial}{\partial \vec{v}_{j}}\right] \times
$$

$$
\left(f_{0 j}+\varepsilon f_{1 j}+\varepsilon^{2} f_{2 j}+\mu \delta f_{h}+\mu \varepsilon \delta f_{l h}+\mu \varepsilon^{2} \Delta f\right)=0
$$

To the order of $\mu$ we have

$$
\hat{L} \delta f_{h}=\frac{e_{j}}{m_{j}} \delta \vec{E}_{h} \cdot \frac{\partial}{\partial \vec{v}_{j}} f_{0 j}
$$

To the order of $\mu \varepsilon$ we have

$$
\hat{L} \delta f_{l h}=\frac{e_{j}}{m_{j}}\left[\vec{E}_{l} \cdot \frac{\partial}{\partial \vec{v}_{j}} \delta f_{l h}+\delta \vec{E}_{h} \cdot \frac{\partial}{\partial \vec{v}_{j}} f_{1 j}+\delta \vec{E}_{l h} \cdot \frac{\partial}{\partial \vec{v}_{j}} f_{0 j}\right]
$$

To the order of $\mu \varepsilon^{2}$ and applying random phase approximation [20] to omit second order quantities we have

$$
\hat{L} \Delta f=\frac{e_{j}}{m_{j}}\left[\vec{E}_{l} \cdot \frac{\partial}{\partial \vec{v}_{j}} \delta f_{l h}+\delta \vec{E}_{l h} \cdot \frac{\partial}{\partial \vec{v}_{j}} f_{1 j}\right]
$$

To obtain different fluctuating parts, using Fourier transform and the method of integration along unperturbed orbit [19] we get after calculation from equation (11)

$$
\begin{aligned}
& \delta f_{h}(\vec{K}, \Omega)=\frac{e_{j}}{m_{j}} \int_{-\infty}^{0} \delta \vec{E}_{h} \cdot \frac{\partial f_{0 j}}{\partial \vec{v}_{j}} e^{\left[i\left\{\vec{K}\left(\vec{r}^{\prime}-\vec{r}\right)-\Omega \tau\right\}\right] d \tau} \\
& =-\sum \frac{e_{j}}{m_{j}} \frac{\delta E_{h}}{\left(K_{\square} v_{j \square}-\Omega\right)} \cdot \frac{\partial f_{0 j}}{\partial v_{j}}
\end{aligned}
$$

and from equation (12)

$$
\begin{aligned}
\delta f_{l h}(\vec{K}-\vec{k}, \Omega-\omega)= & \sum \frac{e_{j}}{m_{j}} \int_{-\infty}^{0}\left[\delta \vec{E}_{l h} \cdot \frac{\partial f_{0 j}}{\partial \vec{v}_{j}}+\delta \vec{E}_{h} \cdot \frac{\partial f_{1 j}}{\partial \vec{v}_{j}}+\delta \vec{E}_{l h} \cdot \frac{\partial f_{h}}{\partial \vec{v}_{j}}\right] \times \\
& e^{\left[i\left\{(\vec{K}-\vec{k}) \cdot\left(\vec{r}^{\prime}-\vec{r}\right)-(\Omega-\omega) \tau\right\}\right] d \tau} \\
= & I_{l h}^{1}+I_{l h}^{2}+I_{l h}^{3}
\end{aligned}
$$

Here

$$
\begin{aligned}
& \int_{-\infty}^{0} e^{\left[i\left\{(\vec{K}-\vec{k}) \cdot\left(\vec{r}^{\prime}-\vec{r}\right)-(\Omega-\omega) \tau\right\}\right] d \tau}=\sum_{s, t} \frac{J_{s}\left(\frac{k_{\perp} v_{\perp}}{\Omega_{j}}\right) J_{t}\left(\frac{k_{\perp} v_{\perp}}{\Omega_{j}}\right) e^{[i(s-t) \theta]}}{i\left\{\left(K_{\square}-k_{\square}\right) v_{j \square}-\left(s \Omega{ }_{j}+\Omega-\omega\right)\right\}} \\
& =\psi_{s, t} \text { (say) }
\end{aligned}
$$

and applying Bessel's Recursion formula 
$J_{n-1}(x)+J_{n+1}(x)=\frac{2 n}{x} J_{n}(x)$

We obtain after calculation

$$
\begin{aligned}
& I_{l h}^{1}=\frac{e_{j}}{m_{j}} \int_{-\infty}^{0} \delta \vec{E}_{l h}(\vec{k}, \omega) \cdot \frac{\partial}{\partial \vec{v}_{j}} \delta f_{0 j} e^{\left[i\left\{(\vec{K}-\vec{k}) \cdot\left(\vec{r}^{\prime}-\vec{r}\right)-(\Omega-\omega) \tau\right\}\right] d \tau} \\
& =-\frac{i e_{j}}{m_{j}} \psi_{s, t}\left[\frac{\delta E_{l h}}{|\vec{K}-\vec{k}|}\left\{-\frac{s \Omega_{j}}{k_{\perp} v_{\perp}} k_{\perp} \frac{\partial}{\partial v_{\perp}}-\left(K_{\square}-k_{\square}\right) \frac{\partial}{\partial v_{\square}}\right\}\right] f_{0 j} \\
& I_{l h}^{2}=\frac{e_{j}}{m_{j}} \int_{-\infty}^{0} \delta \vec{E}_{h}(\vec{k}, \omega) \cdot \frac{\partial}{\partial \vec{v}_{j}} \delta f_{1 h} e^{\left[i\left\{(\vec{K}-\vec{k}) \cdot\left(\vec{r}^{\prime}-\vec{r}\right)-(\Omega-\omega) \tau\right\}\right] d \tau} \\
& =-\frac{i e_{j}}{m_{j}} \psi_{s, t} \delta E_{h} \frac{\partial}{\partial v_{\square}} f_{1 j} \\
& I_{l h}^{3}=\frac{e_{j}}{m_{j}} \int_{-\infty}^{0} \vec{E}_{l}(\vec{k}, \omega) \cdot \frac{\partial}{\partial \vec{v}_{j}} \delta f_{h} e^{\left[i\left\{(\vec{K}-\vec{k}) \cdot\left(\vec{r}^{\prime}-\vec{r}\right)-(\Omega-\omega) \tau\right\}\right] d \tau} \\
& =-\frac{i e_{j}}{m_{j}} \psi_{s, t}\left[\frac{s \Omega_{j}}{k_{\perp} v_{\perp}} E_{l \perp} \frac{\partial}{\partial v_{\perp}}+E_{\square} \frac{\partial}{\partial v_{\square}}\right] \delta f_{h}
\end{aligned}
$$

From equation (13) we have

$$
\begin{aligned}
\Delta f(\vec{K}, \Omega) & =\frac{e_{j}}{m_{j}} \int_{-\infty}^{0}\left(\vec{E}_{l} \cdot \frac{\partial}{\partial \vec{v}_{j}} \delta f_{l h}+\delta \vec{E}_{l h} \cdot \frac{\partial}{\partial \vec{v}_{j}} \delta f_{1 j}\right) e^{[i\langle\vec{K} \cdot(\vec{r}-\vec{r})-\Omega \tau\}] d \tau} \\
& =I^{\prime}+I^{\prime \prime}
\end{aligned}
$$

Where

$$
\begin{aligned}
& I^{\prime}=\frac{e_{j}}{m_{j}} \int_{-\infty}^{0}\left[E_{l \perp} \frac{\partial}{\partial v_{\perp}}+E_{\square \square} \frac{\partial}{\partial v_{\square}}\right] \delta f_{l h} e^{\left[i\left\{K_{\square} \cdot\left(z^{\prime}-z\right)-\Omega \tau\right\}\right] d \tau} \\
& =-\frac{i e_{j}}{m_{j}}\left[E_{l \perp} \frac{\partial}{\partial v_{\perp}}+E_{l \square} \frac{\partial}{\partial v_{\square}}\right] \frac{\delta f_{l h}}{\left(K_{\square} v_{\square}-\Omega\right)} \\
& I^{\prime \prime}=\frac{e_{j}}{m_{j}} \int_{-\infty}^{0} \delta \vec{E}_{l h} \cdot \frac{\partial}{\partial \vec{v}_{j}} f_{1 j} e^{\left[i\left\{K_{\square} \cdot\left(z^{\prime}-z\right)-\Omega \tau\right\}\right] d \tau} \\
& =-\frac{i e_{j}}{m_{j}}\left[\frac{\delta E_{l h}}{|\vec{K}-\vec{k}|}\left\{k_{\perp} \frac{\partial}{\partial v_{\perp}}-\left(K_{\square}-k_{\square}\right) \frac{\partial}{\partial v_{\perp}}\right\}\right] \frac{f_{1 j}}{\left(K_{\square} v_{\square}-\Omega\right)}
\end{aligned}
$$

By using Poisson equation

$$
\vec{\nabla} \cdot \delta \vec{E}_{l h}=-4 \pi n_{j} e_{j} \int f_{l h} d \vec{v}
$$

The expression of modulated field we obtain as

$$
\begin{aligned}
& \delta E_{l h}=-\frac{4 \pi n_{j} e_{j}}{i|\vec{K}-\vec{k}|} \int\left[\frac{i e_{j}}{m_{j}} \psi_{s, t}\left\{-\left(\frac{k_{\perp}}{|\vec{K}-\vec{k}|}\right) \frac{s \Omega j}{k_{\perp} v_{\perp}} \frac{\partial}{\partial v_{\perp}}-\left(\frac{K_{\square}-k_{\square}}{|\vec{K}-\vec{k}|}\right) \frac{\partial}{\partial v_{\square}}\right\} f_{0 j}+\right. \\
& \left.\delta E_{h} \frac{\partial}{\partial v_{\square}} f_{1 j}+\left\{\frac{s \Omega}{k_{\perp} v_{\perp}} E_{l \perp} \frac{\partial}{\partial v_{\perp}}+E_{l \square} \frac{\partial}{\partial v_{\square}}\right\} \delta f_{h}\right] d \vec{v} \\
& =-\frac{\omega_{p j}^{2}}{|\vec{K}-\vec{k}| \square(\vec{K}-\vec{k})} \int \psi_{s, t}\left[\delta E_{h} \frac{\partial}{\partial v_{\square}} f_{1 j}+\left\{\frac{s \Omega_{j}}{k_{\perp} v_{\perp}} E_{l \perp} \frac{\partial}{\partial v_{\perp}}+E_{l \square} \frac{\partial}{\partial v_{\square}}\right\} \delta f_{h}\right] d \vec{v}
\end{aligned}
$$

where

$$
\square(\vec{K}-\vec{k})=1+\frac{\omega_{p j}^{2}}{|\vec{K}-\vec{k}|^{2}} \int \psi_{s, t}\left\{-\frac{s \Omega}{v_{\perp}} \frac{\partial}{\partial v_{\perp}}-\left(K_{\square}-k_{\square}\right) \frac{\partial}{\partial v_{\square}}\right\} \delta f_{0 j} d \vec{v}
$$

To obtain the nonlinear dielectric function of the electrostatic Langmuir wave with frequency $\Omega$ in the presence of the Lower hybrid wave by using Poisson equation

$$
\vec{\nabla} \cdot \delta \vec{E}_{h}=-4 \pi e_{j} n_{j} \int\left[\delta f_{h}+\Delta f\right] d \vec{v}
$$

which takes the form

$\varepsilon_{h}(K, \Omega) \delta E_{h}=0$

The dispersion relation is written as

$\varepsilon_{h}(K, \Omega)=\varepsilon_{0}(K, \Omega)+\varepsilon_{d}(K, \Omega)+\varepsilon_{p}(K, \Omega)$

where $\varepsilon_{0}(K, \Omega)$ is linear part, $\varepsilon_{d}(K, \Omega)$ is direct coupling part and $\varepsilon_{p}(K, \Omega)$ is polarisation coupling part.

Here after calculation we obtain 
$\varepsilon_{0}(K, \Omega)=1+\frac{\omega_{p e}^{2}}{K_{\square}} \int \frac{\frac{\partial f_{0 e}}{\partial v_{\square}}}{K_{\square}-\Omega} d \vec{v}-\frac{\omega_{p i}^{2}}{K_{\square}} \int \frac{\frac{\partial f_{0 i}}{\partial v_{\square}}}{K_{\square}-\Omega} d \vec{v}$

where

$\omega_{p j}^{2}=\frac{4 \pi n_{j} e_{j}^{2}}{m_{j}}, j=e, i$

Considering only dominant parts the expression of $\varepsilon_{d}$ as

$\varepsilon_{d}=-\frac{\omega_{p j}^{2}}{K_{\square}}\left(\frac{e_{j}}{m_{j}}\right)^{2} \int \gamma_{p, q}\left[\left(E_{l \perp} \frac{\partial}{\partial v_{\perp}}+\frac{p \Omega}{K_{\square} v_{\square}} E_{l \square} \frac{\partial}{\partial v_{\square}}\right) \times\right.$

$\left.\psi_{s, t}\left\{\left(E_{l \perp} \frac{\partial}{\partial v_{\perp}}+\frac{s \Omega_{j}}{\left(K_{\square}-k_{\square}\right)} \frac{\partial}{\partial v_{\square}}\right) \delta f_{h}^{\prime}+\frac{s \Omega_{j}}{\left(K_{\square}-k_{\square}\right) v} \frac{\partial}{\partial v_{\square}} f_{1 j}^{\prime}\right\}\right] d \vec{v}$

Where

$\gamma_{p, q}=\sum_{p, q} \frac{J_{p}\left(\frac{K_{\square} v_{\square}}{\Omega_{j}}\right) J_{q}\left(\frac{K_{\square} v_{\square}}{\Omega_{j}}\right) e^{\{i(q-p) \theta\}}}{\Omega-p \Omega_{j}}$

$\delta f_{h}^{\prime}=\sum_{s, t} J_{s}\left(\frac{K_{\square \square}}{\Omega_{j}}\right) J_{t}\left(\frac{K_{\square} v_{\square}}{\Omega_{j}}\right) \frac{e^{\{i(t-s) \theta\}}}{\Omega^{-s \Omega_{j}}}\left(\frac{s \Omega_{j}}{K_{\square \square}}\right) \frac{\partial}{\partial v_{\square}} f_{0 j}(\vec{v})$

$\delta f_{1 j}^{\prime}=\Pi_{a, b}\left(\frac{a \Omega_{j}}{k_{\perp} v_{\perp}} E_{l \perp} \frac{\partial}{\partial v_{\perp}}+E_{l \square} \frac{\partial}{\partial v_{\square}}\right) f_{0 j}(\vec{v})$

and the form of bulky expression of polarization coupling term $\varepsilon_{p}$ as

$\varepsilon_{p}=-\sum \frac{\omega_{p j}^{4}}{\square K_{\square}|\vec{K}-\vec{k}|(\vec{K}-\vec{k})}\left(\frac{e_{j}}{m_{j}}\right)^{2} \quad(A+B) \times(C+D)$

where

$$
A=\int_{\gamma, q}\left[\left(E_{l \perp} \frac{\partial}{\partial v_{\perp}}+\frac{p \Omega_{j}}{K_{\square} V_{\square}} E_{\square \square} \frac{\partial}{\partial v_{\square}}\right)\left\{\psi_{s, t} \frac{1}{|\vec{K}-\vec{k}|}\left(\frac{\partial}{\partial v_{\perp}}-\frac{s \Omega_{j}}{v_{\square}} k_{\square} \frac{\partial}{\partial v_{\square}}\right) f_{0 j}\right\}\right] d \vec{v}
$$

$$
\begin{aligned}
& B=\int \gamma_{p, q} \frac{1}{|\vec{K}-\vec{k}|}\left(-k_{\perp} \frac{\partial}{\partial v_{\perp}}+\frac{p \Omega_{j}}{K_{\square} v_{\square}}\left(K_{\square}-k_{\square}\right) \frac{\partial}{\partial v_{\square}}\right) f_{1}^{\prime} d \vec{v} \\
& C=\int \sum_{p, q} \frac{J_{p}\left(\alpha^{\prime}\right) J_{q}\left(\alpha^{\prime}\right) e^{\{i(q-p)\}}}{\Omega-\omega+k_{\square}-p \Omega_{j}}\left(E_{l \perp} \frac{\partial}{\partial v_{\perp}}+\frac{p \Omega_{j}}{k_{\square} v_{\square}} E_{l \square} \frac{\partial}{\partial v_{\square}}\right) \delta f_{h}^{\prime} d \vec{v} \\
& D=\int \sum_{p, q} \frac{J_{p}\left(\alpha^{\prime}\right) J_{q}\left(\alpha^{\prime}\right) e^{\{i(q-p)\}}}{\Omega-\omega+k_{\square} v_{\square}-p \Omega_{j}} \frac{\partial}{\partial v_{\perp}} f_{1 j}^{\prime} d \vec{v}
\end{aligned}
$$

where

$$
\alpha^{\prime}=\frac{\left(K_{\square}-k_{\square}\right) v_{\square}}{\Omega_{j}}
$$

We consider plasma maser effect for in the Langmuir wave in the presence of low frequency Lower hybrid wave. To estimate the growth rate of Langmuir wave we are using the following expression

$$
\frac{\gamma(\Omega, \vec{K})}{\Omega}=-\frac{\operatorname{Im} \varepsilon_{0}(\Omega, \vec{K})+\operatorname{Im} \varepsilon_{d}(\Omega, \vec{K})+\operatorname{Im} \varepsilon_{p}(\Omega, \vec{K})+\frac{1}{2} \frac{\partial^{2} \varepsilon_{0}}{\partial \Omega \partial t}}{\Omega \frac{\partial \varepsilon_{0}}{\partial \Omega}}
$$

where Im represent the imaginary part of the dielectric function.

By using large argument series of the plasma dispersion function $z\left(\frac{\Omega}{K_{\square} v_{\square}}\right)$ in equation (26) we have

$\varepsilon_{0}(\vec{K}, \Omega)=1-\left(\frac{k_{e}}{K_{\square}}\right)^{2}-\left(\frac{\omega_{p j}}{\Omega}\right)^{2}$

Where $k_{e}$ is the Debye wave number.

After calculation we obtain

$$
\frac{\partial \varepsilon_{0}(\vec{K}, \Omega)}{\partial \Omega}=\frac{2 \omega_{p j}^{2}}{\Omega^{3}}
$$

From previous studies on wave energy upconversion of Langmuir wave in presence of resonant wave through nonlinear wave-particle interaction in homogenous space plasma [18], it was found that the most dominant contribution comes from the polarization coupling term for the growth of nonresonant Langmuir wave. For estimation of growth rate of Langmuir wave in this study on homogeneous 
ionospheric plasma we consider polarization coupling term only.

From equation (28) we have

$$
\operatorname{Im} \varepsilon_{p}=-\sum \frac{\omega_{p j}^{4}}{\square K_{\square}|\vec{K}-\vec{k}|(\vec{K}-\vec{k})}\left(\frac{e_{j}}{m_{j}}\right)^{2}(A \times \operatorname{Im} D)+(C \times \operatorname{Im} D)
$$

Here, due to causality principle $\mathrm{A}$ and $\mathrm{C}$ are reals and $\mathrm{B}$ and $\mathrm{D}$ are complexes.

We consider here the part $(A \times \operatorname{Im} D)$ act as dominant term for the $\operatorname{Im} \varepsilon_{p}$. After integrating by parts and using small argument expansion of Bessel functions and considering dominant terms only we have

$$
A=\frac{2}{K_{\square}\left(K_{\square}-k \square\right)} \frac{1}{c^{3}}\left(\frac{m_{j}}{2 \pi T_{j}}\right)^{\frac{1}{2}}
$$

$\operatorname{Im} D=-\pi \frac{1}{\left|k_{\square}\right| K_{\square}} V_{l h}\left(\frac{m_{j}}{T_{j}}\right)\left(\frac{m_{j}}{2 \pi T_{j}}\right)^{\frac{1}{2}} e^{-\left(\frac{V_{l h}}{v_{e}}\right)^{2}}$

where $v_{l h}$ is the velocity of lower hybrid wave.

After lengthy calculation and taking dominant terms only and considering $\mathrm{j}=\mathrm{e}$ for Langmuir wave, we obtain the approximate expression of growth rate as

$\frac{\gamma_{p}}{\Omega} \square \frac{\sqrt{\pi}}{2}\left(\frac{k_{e}}{k_{\square}}\right)^{2}\left(\frac{k_{\square}}{k_{\perp}}\right)^{2}\left(\frac{v_{e}}{c}\right)^{4}\left(\frac{k_{e}}{k_{\perp}}\right)^{2} \frac{V_{l h}}{v_{e}} e^{-\left(\frac{V_{l h}}{v_{e}}\right)^{2}}$

\section{RESULT AND DISSCUSSION}

From earlier investigations using space based observatories it is found emission of modulated Langmuir wave in the auroral ionosphere, in the solar wind, in the magnetosphere and in space plasma. In the topside auroral ionosphere it was reported parametric decay of Langmuir wave to lower hybrid wave. In our study we consider wave energy upconversion of Langmuir wave through nonlinear wave-particle interaction and obtain its growth rate through plasma maser instability in the presence of lower hybrid wave.

To obtain an estimation of the growth rate of Langmuir wave, we consider the following observational data:

1)The Plasma parameters of topside polar ionosphere according to Freja satellite observations [13]:

Average velocity of electron $v_{e}=3 \times 10^{8} \mathrm{~m} / \mathrm{sec}$

Velocity spread of electron $\Delta v_{e}=2 \times 10^{8} \mathrm{~m} / \mathrm{sec}$

2) Lower hybrid wave parameters in space [21]:

$k_{\perp}=2 \pi \times 10^{-5} \mathrm{~cm} / \mathrm{sec}, k_{\square}=10^{-5} \mathrm{~cm} / \mathrm{sec}$

From calculation the estimated growth rate of Langmuir wave is

$$
\frac{\gamma}{\Omega}=10^{-2}
$$

\section{REFERENCE}

[1] N.L.Davis, Phys.Rev.Lett.; 30; 289; (1974).

[2]V.N.Tsytovich, L.Steno, and H.Wilhemson, Phys. Scripta,11; 251(1975).

[3] M.Nambu,Laser and Particle Beams, 1; 427(1983).

[4]J.Bonnell, J.Kintner, J.E.Wahlund, and J.Holtel, J.Geophys.Res.,102 ; 17; 233 17; 240; (1997).

[5]K.Stasiewicz,B.Holback,V.Krasnoselskish,M.Boehm, and P.Kinter,J.Geophys.Res.,101;21; 51521; 525; (1996).

[6]K.Papadopoulos,andT.Coey,J.Geophys.Res.,79;674(1974).

[7] P.Guio,and F.Forme,Phys.Plasmas, 13; 122902(2006).

[8]Ergun, R.E., C.W. Carlson, J.P. McFadden, J.H. Clemmons, and M.H. Boehm,Geophys. Res. Lett.; 18; 1177 1181; (1991).

[9]C.J.Heinselman, and J.F.Vickrey, J. of Geophys. Res. Space Physics,97; A10; 14905 -14910; (1992).

[10]R.Bingham, D.A.Bryant, and D.S.Hall, Annales Geophysicae 6; 98; (1988).

[11]D.L.Pasmanik,A.G.Demekhov,andV.Y.Trakhtengerts,Radiophys Quantum El $52: 252$ :https : ==doi:org=10:1007=s11141 0099134 y; (2009).

[12]M.C.Kelley, The Earth's Ionospheric Plasma Physics and electrodynamics(Academic Press,Inc.Harcourt Brace Jovanovich Publishers)(2009).

[13]G.V.Lizunov,Y.Khotyantsev, and K.Stasiewicz,J.of Geophys.Res.,106; A11; 24; 755-24; 763; (2001).

[14]P.N.Deka, and A.Borgohain,Physics of Plasmas,18(4); 042311; (2011).

[15]P.N.Deka, and A.Borgohain,J.Plasma Physics,78(5); 515 524; (2012).

[16]M.Singh, and P.N.Deka,Phys.Plasma,12; 102304; (2005).

[17]P. N. Deka and J. K. Deka , J. Plasma Energ . https:// doi.org/10:1007=s10894-018- 0198 - 6; (2018).

[18]P.N.Deka, Ph.D.Thesis, Gauhati University Assam India(1999).

[19] N.A.Krall,A.W.Trivelpiece,Principles of Plasma Physics,(McGraw-Hill Book Company,USA,1973).

[20]R.C.Davidson,Methods in Nonlinear Plasma Theory,Academic Press,New York,(1972).

[21]M.Malingre,R.Pottelette,N.Dobouloz,P.A.Lindqvist,G.Holmgren,and B.Apricio.Geophys.Res.Lett.,19; 1339- 42,(1992). 